\title{
FORCED MOVEMENT OF A BALL IN SPHERICAL CAVITY UNDER KINEMATIC EXCITATION
}

\author{
J. Náprstek ${ }^{*}$, C. Fischer ${ }^{* *}$
}

\begin{abstract}
In the paper the response of a heavy ball rolling inside a semi-spherical cavity under horizontal kinematic excitation is investigated. The system with six degrees of freedom with three non-holonomic constraints is considered. The contact between the ball and the cavity surface is supposed to be perfect without any sliding. The mathematical model using the Appel-Gibbs function of acceleration energy is developed and discussed. The most important post-critical regimes are outlined and qualitatively evaluated on the frequency axis. Numerical experiments have been performed when excitation frequency is slowly swept up and down. Results obtained by means of semi-analytical investigation and numerical simulation are evaluated and physically interpreted. Some applications in civil engineering as a tuned mass damper used on slender structures is outlined. Strengths and weaknesses of solution method are evaluated.
\end{abstract}

Keywords: nonlinear dynamics, non-holonomic systems, Appell-Gibbs formulation, singular states, tuned mass damper

\section{Introduction}

Passive tuned mass dampers (TMD) of various types are widely used in civil engineering. TV towers, masts and other slender structures exposed to wind excitation are usually equipped with such devices. If there is an insufficient place to install a conventional pendulum type device, an absorber of the ball type represents a convenient solution. The first such device in the Czech republic was presented by Pirner (1994) for suppression of the excessive transversal vibrations of a suspended prestressed concrete footbridge over the "Swiss Bay" near Vranov. In this case was the ball intended to be placed in the long curved box and the ball was expected to move in one direction only. The regular ball absorbers woring in both horizontal directions were installed on two TV towers in south-western Bohemia in the late nineties, the technical details are decribed by Pirner and Fischer (2000).

The present paper si devoted to the analysis of the response of a heavy ball rolling inside a semi-spherical cavity under horizontal kinematic excitation. The used mathematical model is based on the Appell-Gibbs apprach, it comprises the system with six degrees of freedom with three non-holonomic constraints. The Appell-Gibbs formulation, see for instance (Pars, 1972; Udwadia, 1998), of a relevant non-holonomic system dynamics proved excellent efficiency in comparison with a conventional way being based on Lagrangian differential system and non-holonomic constraints adjoined via indefinite Lagrange multipliers.

Authors published the governing differential system using both approaches, see (Náprstek, 2013, 2017) and compared their strengths and shortcomings. In the paper (Náprstek, 2017), a number of singular solutions of homogeneous system have been found (no external excitation) for various settings of the non-homogeneous initial conditions and used as limits separating solution groups of a certain character.

This paper is devoted to a system under several types of uni- and bi-axial kinematic excitation of the cavity (non-homogeneous system, homogeneous initial conditions). The slipping-less movement of a ball on a surface is supposed. Two different rolling and spin types of the damping are considered.

Ing. Jiří Náprstek, DrSc.: Institute of Theoretical and Applied Mechanics CAS; Prosecká 76, 19000 Prague 9, tel. +420 286 8821 21; naprstek@itam.cas.cz

** $\quad$ RNDr. Cyril Fischer, Ph.D.: Institute of Theoretical and Applied Mechanics CAS; Prosecká 76, 19000 Prague 9; fischerc@itam.cas.cz 


\section{Governing system}

The Appell-Gibbs approach has been used to carry out the governing differential system modeling movement of a ball inside of a spherical cavity, which is subdued to kinematic excitation in the horizontal plane. For details, see references cited above. The differential system consists of three equation of motion and three equation of non-holonomic constraints:

$$
\begin{aligned}
& J_{s} \dot{\omega}_{x} \quad-u_{C x} \dot{\Omega}_{s}=\left(\left(\ddot{u}_{A y}+\rho\left(\omega_{z} \dot{u}_{C x}-\omega_{x} \dot{u}_{C z}\right)\right)\left(u_{C z}-R\right)-\right. \\
& \left.-u_{C y}\left(g+\rho\left(\omega_{x} \dot{u}_{C y}-\omega_{y} \dot{u}_{C x}\right)\right)\right)-D_{G x} / m, \\
& J_{s} \dot{\omega}_{y} \quad-u_{C y} \dot{\Omega}_{s}=\left(-\left(\ddot{u}_{A x}+\rho\left(\omega_{y} \dot{u}_{C z}-\omega_{z} \dot{u}_{C y}\right)\right)\left(u_{C z}-R\right)+\right. \\
& \left.+u_{C x}\left(g+\rho\left(\omega_{x} \dot{u}_{C y}-\omega_{y} \dot{u}_{C x}\right)\right)\right)-D_{G y} / m, \\
& J_{s} \dot{\omega}_{z}-\left(u_{C z}-R\right) \dot{\Omega}_{s}=\quad\left(\left(\ddot{u}_{A x}+\rho\left(\omega_{y} \dot{u}_{C z}-\omega_{z} \dot{u}_{C y}\right)\right) u_{C y}-\right. \\
& \left.-\left(\ddot{u}_{A y}+\rho\left(\omega_{z} \dot{u}_{C x}-\omega_{x} \dot{u}_{C z}\right)\right) u_{C x}\right)-D_{G x} / m,
\end{aligned}
$$

The meaning of $\dot{\Omega}, J_{s}$ and other quantities is as follows:

$$
\begin{aligned}
\dot{\Omega}_{s}= & u_{C x} \dot{\omega}_{x}+u_{C y} \dot{\omega}_{y}+\left(u_{C z}-R\right) \dot{\omega}_{z}, \\
J_{s}= & \frac{\left(J+m \rho^{2} R^{2}\right)}{m \rho^{2}}, \text { where the numerator represents the mass inertia moment of the ball } \\
& \text { with respect to center of the cavity, } \\
\mathbf{u}_{C}- & \text { displacement vector in contact point } C, \\
\boldsymbol{\omega} & - \text { ball rotation velocity in its center } G, \\
\mathbf{D}_{G}- & \text { damping vector-viscous type (linear functions of) } \boldsymbol{\omega}, \\
\ddot{\mathbf{u}}_{A}- & \text { kinematic excitation in point } A \text { - "southern pole" of the cavity, } \ddot{u}_{A z}=0, \\
R, r- & \text { radius of the cavity and of the ball, respectively, } R>r, \\
g- & \text { gravity constant } \\
\varrho & 1-r / R .
\end{aligned}
$$

It can be shown that $\dot{\Omega}=0$ and therefore the 2 nd column on the left side of the system Eqs (1) can be omitted. Provided we need investigate the response processes in a vertical plane, then only one component remains non-zero and the 2nd horizontal excitation component vanishes as well. In order to obtain the system Eqs (1) in the form with first time derivatives concentrated on left side, the first derivatives $\dot{\mathbf{u}}_{C}$ in its right sides should be expressed in displacements $\mathbf{u}_{C}$ using three non-holonomic constraints:

$$
\begin{aligned}
& \dot{u}_{C x}=\omega_{y}\left(u_{C z}-R\right)-\omega_{z} u_{C y}, \\
& \dot{u}_{C y}=\omega_{z} u_{C x}-\omega_{x}\left(u_{C z}-R\right), \\
& \dot{u}_{C z}=\omega_{x} u_{C y}-\omega_{y} u_{C x} .
\end{aligned}
$$

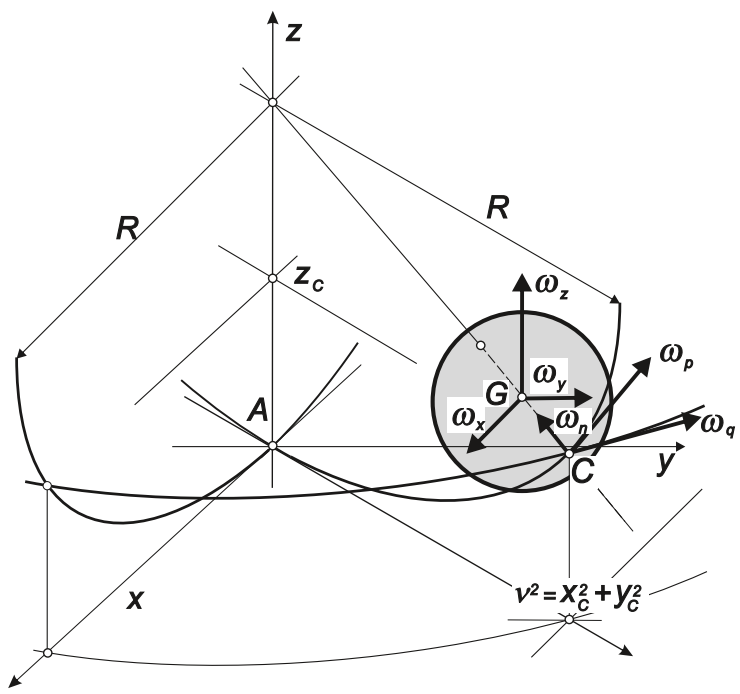

Fig. 1: Moving coordinate system

So we obtained the system of six non-linear ODEs

Eqs $(1,2)$ in normal form with six unknowns functions of time: $u_{C x}, u_{C y}, u_{C z}, \omega_{x}, \omega_{y}, \omega_{z}$. Detailed behavior of the ball as a rotating body is given by angular velocities $\boldsymbol{\omega}$. If the time history of rotation should be traced, then a subsequent run is necessary to obtain rotations by means of Euler angles as solution of the system of three ODEs with an input of angular velocities $\boldsymbol{\omega}$.

\section{Response of the ball under harmonic excitation}

This section is devoted to the case where the cavity undergoes harmonic kinematic excitation in the horizontal plane (only one-direction excitation is reported here).

Two extensive series of tests demonstrate the auto-parametric character of the system. In the first series the response has been evaluated separately for discrete excitation frequencies $\omega$ starting from the homogeneous 

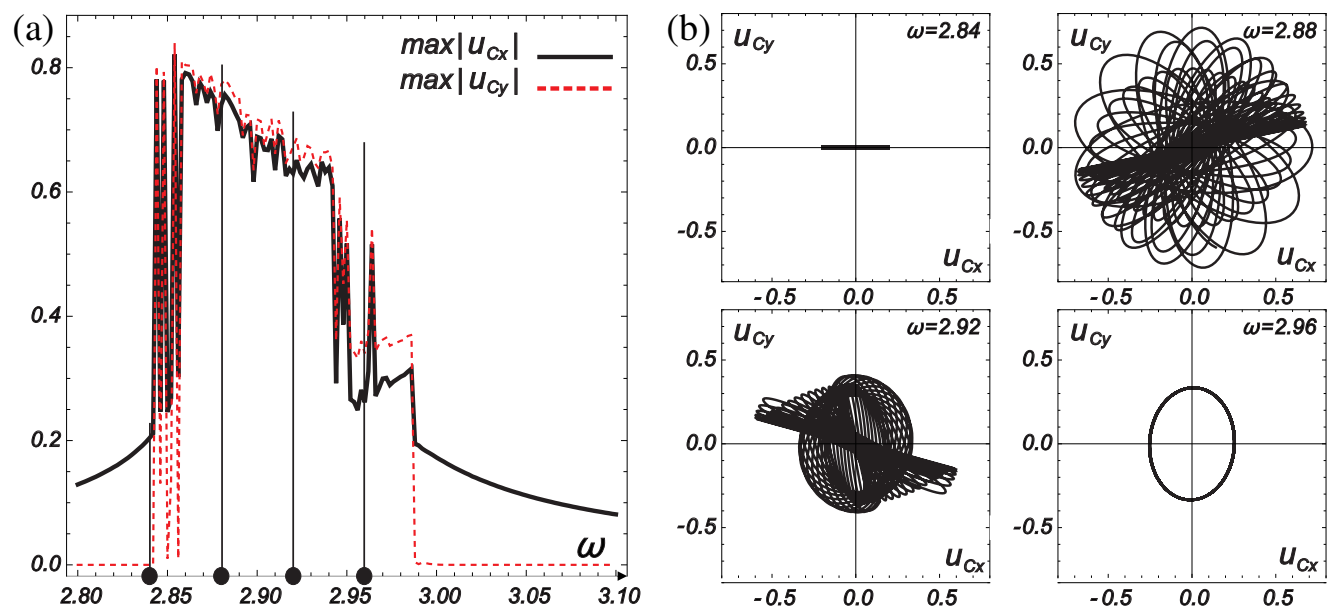

Fig. 2: Response of the ball in the resonance and adjacent zones due to harmonic horizontal excitation of the cavity; (a) amplitude of the displacement as a function of the excitation frequency; $(b)$ vertical views of the ball trajectory for frequencies $\omega=2.84,2.88,2.92,2.96$.
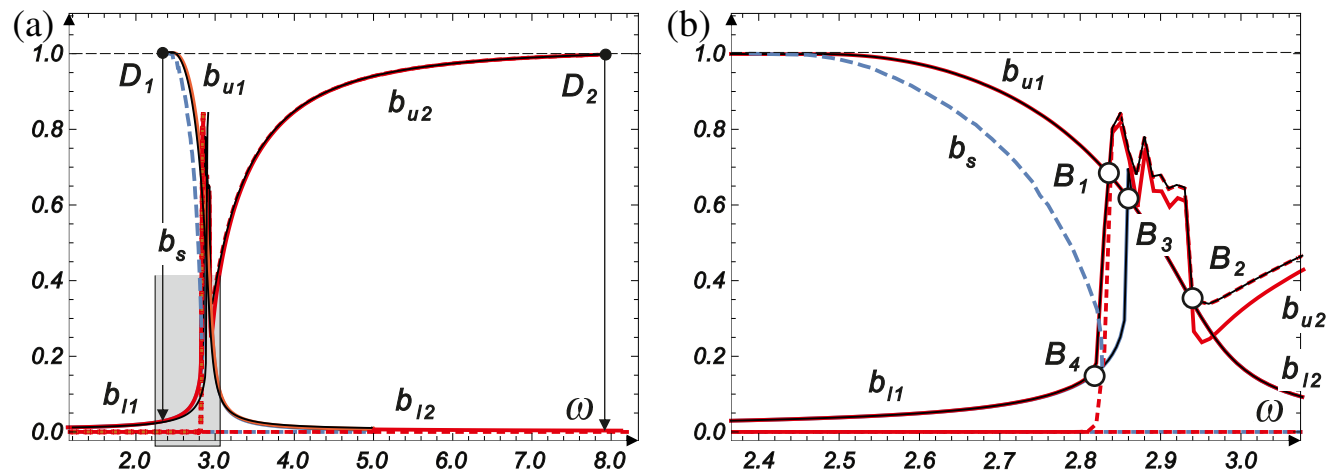

Fig. 3: Amplitudes of the ball displacement under cavity harmonic excitation, when the frequency is swept up and down; (a) amplitudes overview in the interval $\omega \in(1.0,8.0),(b)$ zooming in the interval $\omega \in(2.4,3.1)$; curves: solid red - $\max \left|u_{C x}\right|$, dashed red - $\max \left|u_{C y}\right|$, solid black - absolute displacement amplitude, blue dashed - attraction boundary between $b_{u 1}$ and $b_{l 1}$.

initial conditions. Fig. 2 shows some selected results of numerical simulations which follow from the differential system Eqs $(1,2)$. We briefly point out a couple of features visible in Fig. 2. In the picture (a) we can see the maximal horizontal amplitude of the ball trajectory, when the cavity is kinematically excited in the horizontal plane in $x$ direction. The solid curve represents $\max \left|u_{C x}\right|$ and dashed curve is $\max \left|u_{C y}\right|$ as functions of the exciting frequency $\omega$. We can see that in the interval $\omega \in(0,2.84)$ the semi-trivial solution is stable and so $u_{C y}=0$. The point $\omega=2.84$ is a beginning of the resonance zone, which spans in $\omega \in(2.84,2.99)$, where auto-parametric resonance occurs and amplitudes of both response components are commeasurable. For $\omega>2.99$ the semi-trivial solution is regained. Samples of the trajectory shape are plotted in the picture (b) for four frequencies $\omega=2.84,2.88,2.92,2.96$. Their vertical views demonstrate the character of the semi-trivial and the auto-parametric resonance states. Take a note, that the trajectory since $\omega=2.94$ is a simple ellipse like curve, which does not exhibit any symptom of a chaotic process. Compare this finding with analysis concerning the sweeping up and down excitation frequency for $\omega$ around and above $B_{2}$ bifurcation point (see Fig. 3 and explanation later in this section).

The second series has been controlled by sweeping of the excitation frequency up and down in a large interval and in several detailed regimes in the area of the auto-parametric resonance zone. A few of results are visible in the Fig. 3. The picture (a) demonstrates amplitudes max $\left|u_{C x}\right|$ (solid curves) and $\max \left|u_{C y}\right|$ (dashed curve) and the total amplitude $u_{C r}$ in the interval $\omega \in(1.0,8.5)$. Picture (b) is the magnified detail of the picture (a) within the interval $\omega \in(2.80,3.05)$ in order to make visible the resonance zone. 
Let us pay attention to bifurcation points (BP). The most important reveal $B_{1}$ and $B_{2}$. In the latter one two branches start. The lower one $b_{l 2}$ approaches zero for $\omega \rightarrow \infty$ which indicates the non-moving ball in the vertical view. This branch is takes place in the vertical plane and basically has a form of semi-trivial solution. Its stability increases with rising $\omega>\omega_{B 2}$ as it follows from decreasing negative values of the Lyapunov exponent and of inspection of the relevant stability basins. The upper branch $b_{u 2}$ is spatial. It follows from the resonance zone where the spatial response type has a chaotic character. The relevant attractor reveals as an annular concentric area with diminishing width with increasing $\omega$. The trajectory approaches very quickly a circular form in the horizontal plane. Its level with respect to the vertical axis rises and approaches "equatorial" position. However, the stability of this trajectory decreases and we can see in the Fig. 3, that around $\omega=8.0$ even numerical perturbations of the integration process can overcome the stability limit (despite of very small integration step) and the response trajectory falls down to the lower branch in the point $D_{2}$.

\section{Conclusion}

The presented contribution discuss movement of a heavy ball rolling inside a semi-spherical cavity under horizontal kinematic excitation. This theoretical setup represents a ball type tuned mass damper as an alternative to a pendulum based equipment. The non-linear mathematical model comprises six degrees of freedom with three non-holonomic constraints. The equations of the motion of the ball are derived using the Appel-Gibbs function of acceleration energy. This strategy proved better effectiveness than conventional Lagrangian differential system and non-holonomic constraints adjoined via indefinite Lagrange multipliers. A simple model of the damping mechanism has been incorporated into the Appel-Gibbs system.

The mathematical model is of auto-parametric type. It keeps in full the nonlinear character without any approximations. It was analytically and numerically analyzed with respect to harmonic horizontal excitation. The interval of frequencies leading to instability of the semi-trivial solution was shown and studied in detail. The basic limit cycles were shown and their dangerousness was mentioned. A wide analysis has been performed regarding bifurcation mechanisms and past-bifurcation response branches. Emerging of important limit states has been analyzed, when the excitation frequency is swept up and down. Dynamic stability of individual branches related with increased and dropping excitation frequency has been carefully inspected in the neighborhood of bifurcation points as well as in a far distance from them. It regards also both local and global phenomena of stability keeping, loss and jumping between stable and unstable branches.

\section{Acknowledgement}

The kind support of the Czech Science Foundation project No. 17-26353J and of the RVO 68378297 institutional support are gratefully acknowledged.

\section{References}

Náprstek, J. and Fischer C. (2013) Dynamic response of a heavy ball rolling inside a spherical dish under external excitation. Engineering Mechanics 2013, IT ASCR, Prague, pp 96-106, CD \#46.

Náprstek, J. and Fischer, C. (2017) Non-holonomic dynamics of a ball moving inside a spherical cavity. In: Proc. EURODYN 2017 - 10th Int. Conf. On Structural Dynamics (F. Vestroni et al. eds). Elsevier - Procedia Engineering, Vol. 199, pp 613-618.

Pirner, M. (1994) Dissipation of kinetic energy of large-span bridges. Acta Technica, CSAV, Vol 39, pp 407-418.

Pirner, M. and Fischer, O. (2000) The development of a ball vibration absorber for the use on towers. Jour.Int.Assoc.for Shell and Spatial Structures, Vol 41, No.2, pp 91-99.

Pars, L.A. (1972) A Treatise on Analytical Dynamics (2nd edition). Ox Bow Press, Conn., USA.

Udwadia, F.E. and Kalaba, R.E. (1998) The explicit Gibbs-Appel equation and generalized inverse forms. Quarterly of applied mathematics, Vol 56, No.2, pp 277-288. 